\title{
CRYPTOGENIC STROKE - FROM A HYPOTHESIS TO DEFINITE PROOF. CASE REPORT
}

\author{
Raluca Ileana Nistor \\ Neurology Department, Emergency University Hospital, Bucharest, Romania
}

Current diagnostic standards do not answer all questions regarding the etiology of ischemic stroke. Nowadays, the cryptogenic ischemic stroke (without an obvious cause) still represents $20-30 \%$ of all acute strokes. In the end, this terminology is used in the absence of a definite medical and scientific diagnosis.

The clinical case that will be presented highlights how difficult the differential diagnosis is regarding an ischemic stroke in a young patient, apparently healthy before the acute neurologic event.

A 37 year old male, nonsmoker, obese $(\mathrm{BMI}=40,4)$, with no history of alcohol intake, with only a varicose vein surgical intervention 8 years prior to the current event, complains of sudden onset dizziness. 12 hours before symptom onset, he had sustained an intense physical activity (fitness). Also, he developed rotary vertigo, with nausea and emesis, and he lost the capacity of maintaining orthostatism. The patient was examined in a hospital, were they found: dysarthria, vertigo, horizontal nystagmus, a wide-based gait with lateral veering unsteadiness, but without any localised motor deficit. A CT scan was performed, without any pathological findings. After another episode of vomiting, the patient developed sinus bradycardia which required the administration of $1 \mathrm{mg}$ i.v. atropine. At about 10 hours after the onset of these symptoms, the patient presented in the Neurology Clinic of the University Emergency Hospital Bucharest.

Clinical examination revealed an obese patient, with facial plethora, with $75 \mathrm{bpm}$ and 120/60 $\mathrm{mmHg}$ arterial blood pressure, without any cardiac or vascular murmurs, normal peripheral pulse, with varicose veins, without any signs of deep vein thrombosis; the rest of the clinical examination was normal.

Neurologic examination: the patient was conscious, vigilant and oriented in time and space, with minimal dysarthria (he could speak fluently and coherently), without: vertigo, nystagmus, double vision, tinnitus, hearing or swallowing impairment. Also, the patient presented no meningeal syndrome and no signs of cranial nerve pathology. The patient could walk if someone sustained him, but the gait was ataxic. The Romberg test was negative. He presented minimal dysmetria and right hemiparesis (4/5 MRC scale) with emphasized tendon reflexes on the right side, and absent plantar cutaneous reflex on the same side. The patient had no superficial or profound sensitivity disturbances.

On admission, after the neurological examination, the initial diagnosis was: evolving acute ischemic stroke, after a sustained physical effort, in a young normotensive patient with metabolic syndrome. The first, most probable, diagnosis was considered to be a vertebral artery dissection and arterio-arterial embolism. Of course, other causes of stroke were taken into account, given the patient's age. The emergency ultrasound Doppler of the cervical and cerebral arteries raised the suspicion of a possible left AV dissection (the velocimetric complex was narrowed in the 4th segment of the left vertebral artery, which was also hypoplastic). In the next hours, the patient developed a worsening of the right brachial motor deficit $(3 / 5 \mathrm{BMRc})$ and right central facial palsy. The brain $\mathrm{CT}$ scan and angio CT that were performed 12 hours after symptom onset did not reveal any parenchymal lesions, but also raised the suspicion of a left vertebral ar- 
tery thrombosis in its 4th segment, and confirmed its hypoplastic nature.

In the first hours after admission the patient received aspirin $250 \mathrm{mg} /$ day and statins, but after the worsening of the brachial and crural deficits, i.v. heparin was started, while the aspirin and statin therapy (rosuvastatin $40 \mathrm{mg} /$ day) were continued. During days 5-7 of admission, the transition to oral anticoagulants was made. Despite the lack of the possibility to measure serum homocysteine, folic acid therapy and vitamin B6 and B12 were started given the presence of the metabolic syndrome.

During the seventh day of admission, after the neurologic deficit stabilised, the brain MRI revealed an ischaemic lesion in the left paramedian pons area, the repermeabilisation of the left vertebral artery, without any direct sign of dissection.

The following days, this young patient underwent several investigation procedures, in the search for other biological and imaging evidence that would explain the stroke mechanism in the vertebral territory.

The haematological examination (peripheral blood) was normal. Blood glucose levels (initially $122 \mathrm{mg} / \mathrm{dl}$ ), BUN, creatinine, serum electrolytes (Na, K, Mg), AST, ALT, CK, CKMB, LDH, total cholesterol (126 mg/dl), HDL-C $34 \mathrm{mg} / \mathrm{dl}$, TG 81 $\mathrm{mg} / \mathrm{dl}$, uric acid, total protein levels, cryoglobulins, rheumatoid factor, CRP were normal. Thrombophilia screening revealed a C677T mutation in the MTHFR gene and a heterozygous status for PAI-1 gene deletion; no major risk factor suggesting a severe thrombophilic status were found (ATIII deficit, protein $\mathrm{C}$ and $\mathrm{S}$ deficit, factor $\mathrm{V}$ Leyden, factor II mutation or B group vitamins).

Regarding the cardiovascular examination, the ECG revealed sinus rhythm, HR $78 \mathrm{bpm}$, without repolarisation changes; BP was within normal limits, including ABPM $/ 24 \mathrm{~h}$. The patient had isolated supraventricular premature beats. Transthoracic echocardiography was normal. Transesophageal echocardiography revealed a patent foramen ovale (PFO), with a limited right-to-left shunt at rest, which became significant during the Valsalva maneuver.

Venous ultrasound of the lower extremities ruled out deep vein thrombosis.

Repeated carotid and cerebral arteries ultrasound was normal. Echotomography: hypoplastic left vertebral artery. The rest of the measurements were within normal limits - no atheromatosis, a narrowed velocimetric complex at the V4 level in the left vertebral artery.
Brain CT scan (5 days after symptom onset): recent ischaemic hypodense area in the left parasagittal pons.

Brain MRI ( 7 days after symptom onset): triangular area with diffusion restriction - subacute ischemic stroke in the left parasagittal pons; irregular flow pattern in the V3 segment of the left vertebral artery, with a slight reduction in diameter compared to V2 and V4, without obvious changes in the arterial wall; normal flow in the internal carotid arteries.

In summary, the current case presents a young male patient, with no obvious risk factors (except obesity), which rapidly develops (in hours) an ischaemic stroke manifested by vomiting, nausea, vertigo, astasia abasia, right hemiparesis, 12 hours after intense physical activity during gym. The initial evaluation found: possible thrombotic lesion in the $4^{\text {th }}$ segment of a hypoplastic left vertebral artery and a patent foramen ovale with right-to-left shunt. The brain CT and MRI identified an ischaemic left parasagittal pons lesion. These data suggest an ischaemic stroke of uncertain cause. Taking into consideration the age of the patient, the following possibilities were taken into account (in order of probability): 1. Vertebral artery dissection; 2. Paradoxical embolism through the PFO; 3. Cardioembolic stroke secondary to an atypical source; 4. A hemodynamic factor; 5. The presence of a minor thrombophilia disorder; 6. Intracerebral thrombosis by atherothrombosis.

1) Left vertebral artery dissection on an already hypoplastic artery - was suggested by the occurrence of an ischaemic stroke in a young patient following intense physical activity in the gym. Clinical signs of pons involvement (vertigo, astasia abasia, subsequent hemiparesis) were confirmed by $\mathrm{CT}$ and MRI. Transcranial and cervical Doppler ultrasound confirmed the flow reduction and a modified velocimetric complex secondary to a parietal modification in the V4 segment (there were no visual changes suggestive of a dissection). The patient did not have fluctuant neurologic symptoms, that being usually present in vertebral artery dissection; the clinical examination did not reveal signs of connective tissue disease or vasculitis (EhlersDanlos syndrome, fibromuscular dysplasia or vasculitic lesions). One week after symptom onset the clinical improvement was obvious and the MRI scan did not confirm any parietal change in the vertebral artery (ex. Double lumen), vascular wall hematoma or other signs of dissection). The spontaneous dissection of cerebral arteries is more common in young patients (mean age $42-46$ years) 
and often follows intense physical activities (ex. Sports). It can occur even after minor sport injuries. These patients are frequently hypertensive, obese, have homocysteinemia, connective tissue disease or fibromuscular dysplasia. Cerebral ischaemia can lead to cerebral infarction $(\sim 2 / 3$ of cases) or transient ischaemic attacks. The mechanisms of cerebral ischaemia are either thromboembolic or by hypoperfusion (by general cause or by a modified flow in the dissection area). In conclusion, spontaneous dissection of the left vertebral artery in the V4 segment with subsequent arterio-arterial embolism seems a very likely diagnosis.

2) Paradoxical embolism through a patent foramen ovale (PFO), in this young obese patient, with a history of varicose veins that where surgically treated, needs argumentation. The presence of a PFO with right to left shunt is not a sufficient finding to establish the diagnosis. Patent foramen ovale is a frequent finding during necropsy or transesophageal echocardiography in about $25 \%$ of the general population. Right to left flow through the patent foramen ovale during the Valsalva maneuver was present in this case, but venous Doppler ultrasound of the inferior extremities ruled out recent or older deep vein thrombosis.

The association between a PFO and cryptogenic stroke (or, at least, apparently cryptogenic) is still an unsolved issue. In the classic PICSS trial (Patent Foramen ovale in Cryptogenic Stroke Study), a PFO was found in $38.8 \%$ of patients during transthoracic echocardiography; the septal anomaly was present in $39.7 \%$ of patients with cryptogenic stroke and in $29.9 \%$ of patient with a known cause of ischaemic stroke $(p<0.02)$. On the other hand, 2 major trials (NOMAS - Northern Manhattan Study; Olmstead Study) found no association between the risk of stroke and a patent foramen ovale in young patients or in those older than 60 years.

Some additional factors besides a PFO, seem to increase the risk of paradoxical embolism - the size of the PFO and the presence of an interatrial septal aneurysm - but these are not present in the case of the current patient. A PFO $>4 \mathrm{~mm}$ and a significant shunt seem to raise the risk of a TIA, ischaemic stroke or recurrent stroke. An interatrial septal aneurysm can be an isolated anatomic and echocardiographic anomaly in patients with cryptogenic stroke (about $1.7 \%$ ) but associated with a PFO (9\%); the presence of a concurrent PFO and interatrial septum aneurysm may indicate an increased risk of cardioembolic stroke as opposed to an isolated PFO, probably because of thrombi formation in the aneurysm followed by embolization.
Summing up the clinical, neurological, imaging findings and the arguments in favour and opposed to a possible embolization through a PFO, a cryptogenic embolic stroke cannot be ruled out. The criteria for an unknown source ischaemic stroke are met:

- A non-lacunary stroke detected by CT or MRI

- The absence of intra or extracranial atherosclerosis

- The absence of a major source of cardioembolism

- The absence of other specific identifiable causes of stoke (arteritis, dissection, etc.)

In the current scenario, the most likely embolic source could be deep vein thrombosis of the inferior extremities (obesity, history of varicose veins).

3) Cardioembolic stroke secondary to an atypical source, other than paradoxical embolism this diagnosis is contemplated in any neurological ischaemic event. In the current case, the young age (37 years), the lack of structural heart disease or any suspected or proven supraventricular arrhythmias argue against a cardioembolic stroke. Before ruling out this diagnosis, one must theoretically take into account the existence of asymptomatic atrial tachyarrhythmias (especially recurrent atrial fibrillation) and aortic plaques in the aortic arc as embolic sources. The aorta as a source of embolism can be excluded in this case: there are not clinical or imaging data that suggest atherosclerosis in any major artery (cervical, cerebral, coronary or peripheral) or transesophageal echocardiographic findings (complex aortic plaques or other conditions). Subclinical atrial fibrillation as an embolic source is impossible to exclude in this case. The role of atrial tachyarrhythmias of short duration as a possible source of cryptogenic stroke was intensively studied in recent years. In the CRISTAL-AF trial, which included 441 patients over 40 years of age with a history of cryptogenic stroke, without a history of documented atrial fibrillation, after full clinical assessment and the elimination of other sources of stroke, the patients were assessed for asymptomatic atrial fibrillation. Long term monitoring of atrial arrhythmias by an implantable monitor for 6 months revealed atrial fibrillation in $8.9 \%$ of patient in the cryptogenic stroke arm versus $1.9 \%$ in the control group. After 12 months, asymptomatic short duration atrial fibrillation was found in $12.4 \%$ of patients in the stroke group vs $2 \%$ in the control group. Ischaemic stroke occurred in 5.2\% and 7.1\% of patients at 6 and 12 months compared to $8.6 \%$ and $9.1 \%$ in the control group. Nearly $50 \%$ of the 
atrial fibrillation episodes lasted 12 hours. In another trial (EMBRACE), that included patients with a history of cryptogenic stroke in the last 6 months and no documented atrial fibrillation, 30 day continuous monitoring identified atrial fibrillation five-fold compared to 24-hour monitoring. Short episodes of atrial fibrillation, lasting for several hours, are not proven to be cause thrombosis, but they seem to predict subsequent asymptomatic atrial fibrillation episodes, a risk factor for recurrent ischemic stroke.

In the current setting, after evaluating the cardiovascular and neurological findings, the probability of asymptomatic recurrent atrial fibrillation as a source of isolated vertebral artery embolism is practically minimal. Cardioembolic stroke is usually located in multiple sites (at least on MRI) and is located in the territory of major cerebral arteries. The documentation of atrial fibrillation, even paroxysmal, is mandatory, but not sufficient for the diagnosis of cardioembolic stroke.

4) A contributing hemodynamic factor to stroke occurrence - in the current case, can be discussed. Clinical findings after sustained physical activity, like hypotension, bradycardia, dehydration in the presence of a hypoplastic vertebral artery can suggest a possible hemodynamic contributing factor. Often, cerebral ischemia symptoms in specific hemodynamic conditions are transient and reversible after the restoration of a physiological regional or genereal hemodynamic state. Significant cerebral blood flow reductions can cause localised cerebral ischaemia (cerebral infarction) when concomitant pre-existent vascular lesion are present, which in not the current case.

5) Congenital or acquired thrombophylia - is a possible, but very rare, cause of ischemic stroke; it usually manifests as venous thromboembolism. Thrombophylia mutation screening tests (Factor V Leiden, antithrombin deficit, protein $\mathrm{C}$ and $\mathrm{S}$ deficit, prothrombion 20210 gene mutation, antiphospholypid antibodies) are usually performed in young patients with coronary or cerebral artery thrombosis, that do not present any other obvious prothrombotic disorders. In the current case no major thrombophilic factors where identified, but screening tests revealed a C677T MTHFR gene mutation and PAI-1 gene deletion. The significance of these findings cannot be ignored in the young patient with cryptogenic stroke, at least regarding secondary prevention (folic acid and group B vitamins) and long-term follow-up.

6) Intracranial artery thrombosis secondary to atherosclerosis - is a frequent cause of stroke (in about $5-10 \%$ of cases), and is usually associated with recurrent strokes. It can be considered in this case, given the fact that thrombosis occurred in an nonstenosing vertebral artery lesion.

Often, additional cardiovascular risk factors (hypertension, diabetes, dyslipidaemia, smoking) and other biomarkers (B/A1 apolipoprotein ratio, low levels of adiponectin, PAI-1, etc) are present, as well as atherosclerosis in other vascular territories. These characteristics are not present in the current case. On the other hand, the brain MRI performed 7 days after symptom onset revealed irregular flow in the V4 segment and a slight reduction in vessel diameter (thrombus?), without any vascular wall lesion. Blood flow in the carotid territory was normal. The main hypothetical acute stroke mechanisms in cerebral atherosclerotic lesions are hypoperfusion, arterio-arterial embolism and thrombus extension (these mechanisms can be concurrent).

The other, less common clinical situations like: vasculitis, hypercoagulability syndroms, cardiac embolic diseases, can be ruled out in this case. Therefore, the most likely diagnosis in the presented case, is spontaneous, unique, left vertebral artery dissection with arterio-arterial embolism. There aren't enough arguments to sustain the diagnosis of a cerebral pons infarction caused by paradoxical embolism associated with a PFO. A hemodynamic factor cannot be excluded that contributed to the ischemic cerebrovascular event.

In the first 24 hours, the absolute necessary treatment was represented by antithrombotic therapy: aspirin and anticoagulation with UFH, followed by oral anticoagulation with a VKA. The medication was monitored, well tolerated and efficient. After two weeks, the motor deficit decreased by $>80 \%$, the disarthria disappeared, the right hemiparesis improved and the patient was able to use a walking stick. At one month after the stroke, after rehabilitation treatment, the patient recovered almost completely, and the only deficit was reduced right arm mobility. During the clinical and biological follow-up, there were no new elements to suggest other etiological subtype for the ischemic stroke.

The type and duration of oral anticoagulation is cause for debate. In the case of venous thromboembolism without an identifiable cause, this treatment is indicated for 3 to 6 months. The same period of anticoagulation is indicated in the case of a cervical artery dissection. An extended period of anticoagulation can be considered during patient follow-up, taking into account other prothrombotic factors 
(ex. Thrombophilia). Antivitamin K therapy is the first choice. Novel oral anticoagulants (dabigatran, apixaban, rivaroxaban) have been studied and proven to be efficient and safe for the secondary prevention of cardioembolic stroke in atrial fibrillation. Anticoagulation with rivaroxaban was indicated following evaluation in a foreign center. The exact duration of anticoagulation is to be established.

The presence of several thrombophilia markers in the current situation should not influence the anticoagulation strategy, given the minimal signifi- cance of these mutations in the thrombotic process.

PFO closure does not seem to be indicated in this case. Three major trials (CLOSURI I, RESPECT, PC trial) studied the efficiency and safety of PFO closure compared to optimal medical therapy and concluded that PFO closure is not superior compared to optimal medical treatment in preventing recurrent stroke or transient ischaemic events.

Besides oral anticoagulation (rivaroxaban), the patient was advised to lose weight and was prescribed statins, folic acid and group B vitamins. The patient will be followed-up regularly.

\section{REFERENCES}

1. Smith V.S., English F.D., Johnston S.C. Cerebro-vascular diseases - Harrison's Neurology in Clinical Medicine - Ed. Hausers, McGraw Hill, 2010.

2. Kaksman Z., Klein G. Cryptogenic stroke. Card. Electrophysiol Clin 2014, 6, 161-167

3. Hamma S., Sacco R.L. Patent foramen ovale and stroke. Circulation 2005, 112, 1063-72.

4. Kitsios G.D., Dahabreh J.J. et al. Patent foramen ovale closure and medical treatment for secondary stroke prevention: a systematic review of observational and randomized evidence. Stroke 2012, 43: 422-431.

5. Hast R.G., Diener H.C., Coutts S.B. et al. Embolic strokes of undetermined source: the case for a new clinical construct. Lancet Neurol. 2014, 13: 429-38.

6. Sanna T., Diener H.C., Passman R.S. et al. - for the CRISTAL-AF. Cryptogenic stroke and atrial fibrillation. N Engl J Med 2014, 370: 2478-86.

7. Gladstone D.J., Spring M., Dorian P. et al. For the EMBRANCE Investigators and Coordinators. Atrial fibrillation in patients with cryptogenic stroke. N Engl J Med 2014, 270: 2467-77.

8. Carroll J.D., Saver J.L., Thaler D.E. et al. for the RESPECT Investigators. Closure of patent foramen ovale versus medical therapy after cryptogenic stroke. N Engl J Med 2013, 368: 1092-100.

9. Cotter P.E., Martin P.J., Ring L. et al. Incidence of atrial fibrillation detected by implantable loop recorders in unexplained stroke. Neurology 2013, 80: 1546 - 50.

10. Qurehi A.I., Laplan L.R. Intracranian atherosclerosis. Lancet 2014, 383, 984-99. 